\title{
Effects of phenotypes in heterocyclic aromatic amine (HCA) metabolism-related genes on the association of HCA intake with the risk of colorectal adenomas
}

Barbir, Aline ; Linseisen, Jakob ; Hermann, Silke ; Kaaks, Rudolf ; Teucher, Birgit ; Eichholzer, Monika ; Rohrmann, Sabine

\begin{abstract}
BACKGROUND: Heterocyclic aromatic amines (HCA), formed by high-temperature cooking of meat, are well-known risk factors for colorectal cancer (CRC). Enzymes metabolizing HCAs may influence the risk of CRC depending on the enzyme activity level. We aimed to assess effect modification by polymorphisms in the HCA-metabolizing genes on the association of HCA intake with colorectal adenoma (CRA) risk, which are precursors of CRC. METHODS: A case-control study nested in the EPIC-Heidelberg cohort was conducted. Between 1994 and 2005, 413 adenoma cases were identified and 796 controls were matched to cases. Genotypes were determined and used to predict phenotypes (i.e., enzyme activities). Odds ratios (OR) and corresponding $95 \%$ confidence intervals (CI) were calculated by logistic regression analysis. RESULTS: CRA risk was positively associated with PhIP, MeIQx, and DiMeIQx ( $\mathrm{p}$ trend $=0.006,0.022$, and 0.045, respectively) intake. SULT1A1 phenotypes modified the effect of MeIQx on CRA risk (p (Interaction) > 0.01) such that the association of MeIQx intake with CRA was stronger for slow than for normal phenotypes. Other modifying effects by phenotypes did not reach statistical significance. CONCLUSIONS: HCA intake is positively associated with CRA risk, regardless of phenotypes involved in the metabolizing process. Due to the number of comparisons made in the analysis, the modifying effect of SULT1A1 on the association of HCA intake with CRA risk may be due to chance.
\end{abstract}

DOI: https://doi.org/10.1007/s10552-012-0017-8

Posted at the Zurich Open Repository and Archive, University of Zurich

ZORA URL: https://doi.org/10.5167/uzh-69175

Journal Article

Published Version

Originally published at:

Barbir, Aline; Linseisen, Jakob; Hermann, Silke; Kaaks, Rudolf; Teucher, Birgit; Eichholzer, Monika; Rohrmann, Sabine (2012). Effects of phenotypes in heterocyclic aromatic amine (HCA) metabolismrelated genes on the association of HCA intake with the risk of colorectal adenomas. Cancer Causes Control, 23(9):1429-1442.

DOI: https://doi.org/10.1007/s10552-012-0017-8 


\title{
Effects of phenotypes in heterocyclic aromatic amine (HCA) metabolism-related genes on the association of HCA intake with the risk of colorectal adenomas
}

\author{
Aline Barbir · Jakob Linseisen - Silke Hermann • \\ Rudolf Kaaks • Birgit Teucher • Monika Eichholzer • \\ Sabine Rohrmann
}

Received: 21 February 2012/ Accepted: 13 June 2012/Published online: 28 June 2012

(C) Springer Science+Business Media B.V. 2012

\begin{abstract}
Background Heterocyclic aromatic amines (HCA), formed by high-temperature cooking of meat, are wellknown risk factors for colorectal cancer (CRC). Enzymes metabolizing HCAs may influence the risk of CRC depending on the enzyme activity level. We aimed to assess effect modification by polymorphisms in the HCAmetabolizing genes on the association of HCA intake with colorectal adenoma (CRA) risk, which are precursors of CRC.

Methods A case-control study nested in the EPIC-Heidelberg cohort was conducted. Between 1994 and 2005, 413 adenoma cases were identified and 796 controls were matched to cases. Genotypes were determined and used to predict phenotypes (i.e., enzyme activities). Odds ratios (OR) and corresponding $95 \%$ confidence intervals (CI) were calculated by logistic regression analysis.

Results CRA risk was positively associated with PhIP, MeIQx, and DiMeIQx ( $p$ trend $=0.006,0.022$, and 0.045, respectively) intake. SULTIAl phenotypes modified the effect of MeIQx on CRA risk ( $\left.p_{\text {Interaction }}>0.01\right)$ such that the association of MeIQx intake with CRA was stronger for
\end{abstract}

\footnotetext{
A. Barbir · M. Eichholzer · S. Rohrmann ( $\varangle)$

Division of Cancer Epidemiology and Prevention, Institute of Social and Preventive Medicine, University of Zurich, Hirschengraben 84, 8001 Zürich, Switzerland

e-mail: sabine.rohrmann@ifspm.uzh.ch

\section{J. Linseisen}

Institute of Epidemiology I, Helmholtz Zentrum München, Neuherberg, Germany

J. Linseisen · S. Hermann · R. Kaaks · B. Teucher .

S. Rohrmann

German Cancer Research Center (DKFZ), Heidelberg, Germany
}

slow than for normal phenotypes. Other modifying effects by phenotypes did not reach statistical significance.

Conclusions HCA intake is positively associated with CRA risk, regardless of phenotypes involved in the metabolizing process. Due to the number of comparisons made in the analysis, the modifying effect of SULTIAl on the association of HCA intake with CRA risk may be due to chance.

Keywords Colorectal adenoma $\cdot$ Genetic polymorphisms . Phenotypes $\cdot$ Heterocyclic aromatic amines

\section{Introduction}

Colorectal cancer (CRC) is the third most common cancer in the world; in Europe, it ranges on the second place [1]. Studies have shown an increased CRC risk with high intake of red or processed meat or meat cooked at high temperature [2-4]. Heterocyclic aromatic amines (HCA) are formed during meat preparation at high temperature $\left(>130{ }^{\circ} \mathrm{C}\right)$ due to a chemical reaction from amino acids, creatinine or creatine, and sugar [5]. The amount of HCA depends on meat type, cooking time, and meat preparation like grilling, frying, or barbecuing. PhIP (2-amino-1methyl-6-phenylimidazo[4,5-b]pyridine), MeIQx (2-amino3,8-dimethylimidazo[4,5-f]quinoxaline), and DiMeIQx (2amino-3,4,8-dimethylimidazo[4,5-f]quinoxaline) are the most common HCAs found in nutrition [6, 7].

A systematic review reported that most epidemiological studies observed a positive association of HCA intake with CRC [8]. The carcinogenicity of HCAs is also well known from animal studies $[9,10]$. In contrast, results concerning colorectal adenomas (CRA), which are well-established precursors of CRC $[8,11]$, are inconsistent; up to date a 
dozen studies examined the association of particular HCAs (PhIP, MeIQx, and DiMeIQx) with the risk of CRA [1223]. In our previous study [21], for example, PhIP intake was associated with an increased relative risk of 1.47 (95\% CI: 1.13-1.93, 4th vs. 1st quartile) with colorectal adenoma, whereas no associations were observed for MeIQx and DiMeIQx. The conflicting results may in part be explained by genetic variation in the metabolism of HCAs.

Heterocyclic aromatic amines have to be metabolized by enzymes to become carcinogenic. They need bioactivation from phase I and phase II enzymes [24]. Firstly, HCAs are transported to the liver and hydroxylated into $N$-hydroxylamine by cytochrome P450 1A2 (CYP1A2) [25, 26]. The resulting $N$-hydroxyl ion is transported to the colon mucosa, where it is $\mathrm{O}$-acetylated by $\mathrm{N}$-acetyltransferase 1 (NAT1) and $N$-acetyltransferase 2 (NAT2) or sulfotransferase (SULT). $N$-Acetoxyamin is formed and transported to the target organ, where reactive nitrenium ions are formed, which can react with DNA and ultimately result in CRA. Other enzymes help detoxifying HCAs, such as glutathione S-transferases (GST) or UDP-glucuronyltransferases (UGT) $[4,26]$. We have previously observed some effects of genetic polymorphisms on CRA risk [27]. According to the HCA-metabolizing process, interactions of fast activating and slow detoxifying HCA-metabolizing phenotypes may increase the risk of CRA in association with HCA intake. Vice versa, the combination of slow activating and fast detoxifying HCA-metabolizing phenotypes may decrease the association of HCA intake with the CRA risk.

There are only a limited number of studies looking at genetic polymorphisms with respect to the association of HCA intake with the CRA risk [13, 14, 19, 23, 28]. Most of these studies considered only a few of the relevant polymorphisms.

In this study, we evaluated the modifying effect of genetic polymorphisms from 7 enzymes involved in the HCA metabolism (CYP1A2, NAT1, NAT2, SULT1A1, GSTA1, UGT1A7, and UGT1A9) on the association of HCA with CRA risk, setting the focus on phenotypes that were predicted from genotypes.

\section{Methods}

Study design and population

The European Prospective Investigation into Cancer and Nutrition (EPIC) is a large European-wide prospective cohort study investigating the etiology of cancers with detailed information on lifestyle factors, diet, and biomarkers. Over half a million people were recruited in 10
European countries. In Heidelberg and surrounding areas, 25,546 participants aged 35-64 were enrolled at random from 1994 to 1998. Biological specimens were collected from 24,235 participants [29]. Regular follow-ups are conducted every 2-3 years with the aim to gain information on incident diseases, in particular cancer, including prelesions such as CRA. Other baseline characteristics, recruitment procedures, of the population-based study are reported elsewhere [30]. All participants signed a consent form at baseline, and the ethical committee of the Heidelberg Medical School has approved the current study.

A nested case-control study was conducted within EPIC-Heidelberg among 5,064 participants who had stated in the follow-up that they had had a colonoscopy. Among those, we identified 536 adenoma cases between start of recruitment (1994) and December 2005. CRA was verified and coded relying on the 2 nd version of the International Classification of Diseases for Oncology (ICD-O2). Four hundred and forty-four cases were included in the study after exclusion of prevalent and incident cases of cancer (except non-melanoma skin cancer), stroke, or myocardial infarction. For each case, 2 controls matched by age at colonoscopy ( \pm 1 year), sex, and recruitment year $( \pm 0.5$ year) were selected among those who had had a negative colonoscopy because adenomas are known to be quite often undetected for a long time and who were free of cancer, stroke, and myocardial infarction. Due to missing information on HCA intake or miscarried genotyping, our case-control set finally consisted of 413 cases and 796 controls.

\section{Assessment of HCA exposure}

Dietary intake was assessed by a validated 158 -item food frequency questionnaire (FFQ) at baseline [31] based on self-evaluation. Detailed information on meat consumption and preparation during the last 12 months was collected. In the second follow-up (2001-2003), participants additionally provided information on the degree of browning, based on pictures showing meat lightly, moderately, strongly or extreme browned, and preferred cooking methods for meat [32]. Due to this supplementary information, the intake of the most abundant HCAs (PhIP, MeIQx, DiMeIQx) was calculated for the EPIC-Heidelberg cohort, relying on published data $[33,34]$.

Laboratory analyses

Genomic DNA was extracted from buffy coats with FlexiGene kit (Qiagen Hilden, Germany) in accordance with the manufacturer's instructions. Genotyping was performed at Bioglobe (Hamburg, Germany). CYP1A2 (A-164C, rs762551), NAT1 (C1095A, rs15561), NAT2 (T341C, 
rs1801280; G590A, rs1799930), SULT1A1 (G638A, rs928 2861), GSTA1 (G-52A, rs3957357), UGT1A7 ( $>$ G , rs17 868323; G > A, rs17868324; T > C, rs11692021), and $U G$ T1A9 (A(T)9/10AT, rs3832043) genotypes were determined as multiplex on the MassArray system (Sequenom, San Diego, USA) applying the iPLEX method and matrixassisted laser desorption/ionization (MALDI-TOF). Interexperimental reproducibility and accuracy was verified by $8 \%$ of duplicated samples presenting concordant genotype results. Genotyping of NAT1 (T1088A, rs1057126; G560A, rs4986782) and NAT2 (G857A, rs1799931) was performed in Heidelberg using a LightCycler 480 (Roche, Mannheim, Germany) hybridization technology with real-time polymerase chain reaction (PCR) and melting curve analysis. Determination was carried out in triplicate and a SD of $>10 \%$ led to repeated analysis. Quality control was done by repeating analyses for $5 \%$ of the samples, resulting in accordance with $>95 \%$ of the assigned genotypes. All laboratory analyses were carried out with the laboratory personnel blinded to the case-control status.

\section{Statistical analyses}

In $C Y P 1 A 2$, the $C Y P 1 A 2 * 1 F$ polymorphism (rs762551) A-164C was determined. The homozygous carriers of the A allele were classified as having normal enzyme activity, whereas carriers of the $\mathrm{CA}$ or $\mathrm{CC}$ alleles as having rapid activity [35]. In NAT1, 3 SNPs were determined: T1088A (rs1057126), G560A (rs4986782), and C1095A (rs15561) to classify the $N A T 1 * 4$ (wild type), NAT1*10 (T1088A and C1095A), NAT1*11 (C1095A), and NAT1*14 allele (T1088A, C1095A, and G560A). For NAT1, no phenotypes were predicted due to controversial information on the phenotypic enzyme activity in the literature. Therefore, genotypes were presented, whereas $* 10 / * 10$ alleles and $* 10 / *$ others alleles were pooled into one group, due to the small number in each group. Three SNPs were evaluated in NAT2 (wild type $N A T 2 * 4$ allele) [T341C (NAT2*5, rs1801280), G590A (NAT2*6, rs1799930), and G857A $(N A T 2 * 7$, rs1799931)] to predict two phenotypes: Rapid acetylators were defined as carriers of at least one $N A T 2 * 4$ allele $(* 4 / * 4, * 4 / * 5, * 4 / * 6, * 4 / * 7)$ and all other combinations as slow phenotypes $(* 5 / * 5, * 5 / * 6, * 5 / * 7, * 6 / * 6, * 6 /$ $* 7, * 7 / * 7)$.

Three SNPs in UGT1A7 [T387G (rs17868323, Asp129Lys), G392A (rs17868324, Arg131Lys), and T622C (rs11692021, Trp208Arg)] were used to identify carriers of the UGT1A7*1 allele (wild type), the UGT1A7*2 allele (Asp129Lys and Arg131Lys), the UGT1A7*3 allele (Asp129Lys, Arg131Lys, and Trp208Arg) and the UGT1A7*4 allele (Trp208Arg). These four alleles were used to determine phenotypes with slow $(* 3 /$ $* 3, * 3 / * 4, * 4 / * 4)$, intermediate $(* 1 / * 3, * 1 / * 4, * 2 / * 3, * 2 / * 4)$, and rapid enzyme activity $(* 1 / * 1, * 1 / * 2, * 2 / * 2)$ [36]. SULT1A1 (rs9282861) enzyme activity was defined as normal activity with carriers of the $\mathrm{GG}(* 1 / * 1)$ and $\mathrm{GA}(* 1 / * 2)$ alleles, while the AA $(* 2 / * 2)$ alleles were defined as having slow activity [37]. For GSTA1 (rs3957357), GG or GA alleles result in normal activity and AA in slow activity [38]. For UGT1A9, individuals with deletions at rs3832043 (A(T)9AT) were classified as having normal activity, whereas DEL/T or TT ((T)10AT) variants are leading to rapid activity [39]. An overview of the predicted phenotypes is given in Table 1.

Firstly, the association of HCA intake with the CRA risk was calculated using conditional logistic regression stratified by case set. PhIP, MeIQx, and DiMeIQx were stratified into quartiles based on the distribution in controls. Confounders were selected due to known results from literature: history of familial colon cancer (yes/no), smoking status (never, former, or current), pack-years of smoking, regular use of non-steroidal anti-inflammatory drugs (NSAID) (yes/no), waist circumference, alcohol consumption, and fat intake. Inclusion of variables other than the aforementioned confounders did not materially influence the results (e.g., physical activity, BMI, fruit intake, fiber intake, energy intake). Secondly, we evaluated potential effect modification of the association between the intake of HCAs and the risk of CRA by selected polymorphisms. Therefore, we calculated OR (and $95 \% \mathrm{CI}$ ) of CRA for HCA quartiles stratified by phenotype with unconditional logistic regression adjusting for sex and age (i.e., matching factors) and additionally for the confounders mentioned above (multivariable adjustment). Tests for interaction were done using the cross-product terms of HCA quartiles and the selected phenotypes. The effect modification and correspondent test for interaction was also calculated with HCAs as continuous variables. Thirdly, we were interested in high-risk phenotype group vs. low-risk phenotype group. Thus, we built groups of enzymes that possibly accelerate the progress of developing CRA (high activating and slow detoxifying HCA enzymes) and groups of enzymes, which may reduce the development of CRA (slow activating and high detoxifying HCA enzymes). At least 4 of the 7 enzymes had to be in the according phenotype group. A third group included all remaining enzyme combinations. Low-CRA-risk group was defined when at least four of seven following phenotypes were available: rapid CYP1A2, NAT1*10/*10 or NAT1*10/other, rapid NAT2, intermediate SULT1A1, slow GSTA1, slow UGT1A7, and normal UGT1A9. HighCRA-risk group was defined when at least four of seven following phenotypes were available: normal CYP1A2, NAT $1 * 4 / * 4$, slow NAT2, slow SULT1A1, normal GSTA1, rapid UGT1A7, and rapid UGT1A9. The remaining enzyme combinations were assigned to the intermediateCRA-risk group. The effect of these enzyme groups on the association of HCA intake with the risk of CRA was 
Table 1 Overview of genotypes and predicted phenotypes

\begin{tabular}{|c|c|c|c|}
\hline Enzyme & Function of the enzyme & Polymorphism site & Phenotype (enzyme activity) \\
\hline CYP1A2 & Phase I enzyme & Wild type & Normal activity $(* 1 \mathrm{~A} / * 1 \mathrm{~A})$ \\
\hline CYP1A2*1F & & $\mathrm{A}^{-164} \mathrm{C}($ rs 762551$)$ & Rapid activity $(* 1 \mathrm{~A} / * 1 \mathrm{~F}, * 1 \mathrm{~F} / * 1 \mathrm{~F})$ \\
\hline $\mathrm{NAT} 1 * 4$ & Phase II enzyme & Wild type & No phenotypes predicted, but building of 3 groups: \\
\hline NAT $1 * 10$ & & $\begin{array}{l}\mathrm{T}^{1088} \mathrm{~A}(r s 1057126), \\
\mathrm{C}^{1095} \mathrm{~A}(r s 947894)\end{array}$ & Group 1: $(* 4 / * 4)$ \\
\hline NAT1 $* 11$ & & $\mathrm{C}^{1095} \mathrm{~A}(r s 15561)$ & Group 2: $(* 10 / * 10, * 10 / *$ other $)$ \\
\hline NAT $1 * 14$ & & $\begin{array}{l}\mathrm{T}^{1088} \mathrm{~A}(r s 1057126), \\
\mathrm{C}^{1095} \mathrm{~A}(r s 947894), \\
\mathrm{G}^{560} \mathrm{~A}(r s 4986782)\end{array}$ & Group 3: (*other/*other) \\
\hline NAT $2 * 4$ & Phase II enzyme & Wild type & Normal metabolizer $(* 4 / * 4, * 4 / * 5, * 4 / * 6, * 4 / * 7)$ \\
\hline NAT2 $* 5$ & & $\mathrm{~T}^{341} \mathrm{C}($ rs 1801280$)$ & $\begin{array}{l}\text { Slow metabolizer }(* 5 / * 5, * 5 / * 6, * 5 / * 7, * 6 / * 6, * 6 / \\
\quad * 7, * 7 / * 7)\end{array}$ \\
\hline NAT $2 * 6$ & & $\mathrm{G}^{590} \mathrm{~A}(r s 1799930)$ & \\
\hline NAT $2 * 7$ & & $\mathrm{G}^{857} \mathrm{~A}(r s 1799931)$ & \\
\hline SULT1A1*1 & Phase II enzyme & Wild type & Normal activity $(* 1 / * 1, * 1 / * 2)$ \\
\hline SULT1A $1 * 2$ & & $\mathrm{G}^{638} \mathrm{~A}(r s 9282861)$ & Slow activity $(* 2 / * 2)$ \\
\hline GSTA $1 * \mathrm{~A}$ & Phase II enzyme & Wild type & Normal activity $\left(* \mathrm{~A} / * \mathrm{~A}, \mathrm{~A} /{ }^{*} \mathrm{~B}\right)$ \\
\hline GSTA $1 * \mathrm{~B}$ & & $\mathrm{C}^{-69} \mathrm{~T}($ rs 3957357) & Slow activity $\left(* \mathrm{~B} / *^{*} \mathrm{~B}\right)$ \\
\hline UGT1A7*1 & Phase II enzyme & Wild type & Rapid activity $(* 1 / * 1, * 1 / * 2, * 2 / * 2)$ \\
\hline UGT1A7*2 & & $\begin{array}{l}\operatorname{asp}^{129} \text { lys }\left(\mathrm{T}^{387} \mathrm{G}, r s 17868323\right) \\
\arg ^{131} \text { lys }\left(\mathrm{G}^{392} \mathrm{~A}, r s 17868324\right)\end{array}$ & Intermediate activity $(* 1 / * 3, * 1 / * 4, * 2 / * 3, * 2 / * 4)$ \\
\hline UGT1A7*3 & & $\begin{array}{l}\operatorname{asp}{ }^{129} \text { lys }\left(\mathrm{T}^{387} \mathrm{G}, r s 17868323\right), \\
\arg ^{131} \text { lys }\left(\mathrm{G}^{392} \mathrm{~A}, r s 17868324\right), \\
\operatorname{trp}^{208} \arg \left(\mathrm{T}^{622} \mathrm{C}, r s 11692021\right)\end{array}$ & Slow activity $(* 3 / * 3, * 3 / * 4, * 4 / * 4)$ \\
\hline UGT1A7*4 & & $\operatorname{trp}^{208} \arg \left(\mathrm{T}^{622} \mathrm{C}, r s 11692021\right)$ & \\
\hline UGT1A9*1 & Phase II enzyme & Wild type, $\mathrm{A}(\mathrm{T})_{9} \mathrm{AT}$ & Normal activity $(* 1 / * 1)$ \\
\hline UGT1A9*22 & & $\mathrm{A}(\mathrm{T})_{10} \mathrm{AT}(r s 3832043)$ & Rapid activity $(* 22 / * 22, * 1 / * 22)$ \\
\hline
\end{tabular}

calculated with unconditional logistic regression, whereas the reference categories consisted of participants in the lowest quartile of HCAs. Finally, a sensitivity analysis was conducted to evaluate the effect of the late implementation on the food questionnaire; that is, we excluded all cases that were diagnosed before the meat preparation questionnaire was applied ( $n=228$ ). Two-sided $p<0.05$ was considered statistically significant. All statistical analyses were conducted using STATA version 11.

\section{Results}

Table 2 shows the baseline characteristics of the study population. Cases were more likely to be former or current smokers than controls and had a higher level of pack-years of smoking than controls. Additionally, family history of colon cancer and alcohol consumption was more common in cases than in controls. Neither BMI nor waist circumference differed significantly between cases and controls. Education level was distributed similarly between the two groups. No statistically significant differences were observed for physical activity, NSAID use at baseline and fat intake between cases and controls. Median PhIP, MeIQx, and DiMeIQx intake were higher in cases than in controls.

There was an elevated risk of CRA with increasing PhIP, MeIQx, and DiMeIQx intake, which remained statistically significant after adjusting for confounders ( $p$ trend $=0.006,0.022,0.045$, respectively; Table 3 ). A sensitivity analysis including only cases diagnosed after applying the 2nd FFQ did not reveal results materially different from results of the complete case group.

SNPs in 7 genes of phase I or phase II carcinogenmetabolizing enzymes were analyzed. As reported earlier [27], main effects of genotypes on CRA risk were restricted to SNPs in NAT1 (rs15561 and rs1057126; rarer alleles were inversely associated with CRA risk, but no statistically significant effects were observed for predicted phenotypes), NAT2 (combined genotype: predicted medium (vs. slow) acetylator phenotype with a decreased risk of CRA), and GSTAl (rs3957357; predicted decreased 
Table 2 Baseline characteristics of cases and controls in the case-control study nested in the European Prospective Investigation into Cancer and Nutrition (EPIC)-Heidelberg study

$\begin{array}{lll}\text { Cases }(n=413) & \text { Controls }(n=796) & p^{\mathrm{a}} \\ n(\%)^{\mathrm{b}} & n(\%)^{\mathrm{b}}\end{array}$

Colorectal adenoma characteristics

Adenoma location

$\begin{array}{lr}\text { Right colon } & 114(27.6) \\ \text { Left colon } & 149(36.1) \\ \text { Rectum } & 93(22.5) \\ \text { Missing } & 57(13.8) \\ \text { General characteristics } & \end{array}$

General characteristics

Age at baseline, mean $\pm \mathrm{SD}$

$54.5( \pm 6.20)$

$270(34.2)$

$143(34.1)$

Females

BMI males, mean \pm SD

BMI females, mean \pm SD

Waist circumference males $(\mathrm{cm})$, mean \pm SD

Waist circumference females $(\mathrm{cm})$, mean $\pm \mathrm{SD}$

Education

None or primary school

Technical or professional school

Secondary school

University degree

Smoking status

Never

Former

Current

Pack-years of smoking, mean \pm SD

Physical activity

Inactive

Moderately inactive

Moderately active

Active

NSAID (use at baseline)

Alcohol intake $(\mathrm{g} / \mathrm{d})$, mean $\pm \mathrm{SD}$

Fat intake (g/d), mean \pm SD

Family history of colon cancer

Intake of heterocyclic aromatic amines (ng/d)

PhIP, median (interquartile range)

MeIQx, median (interquartile range)

DiMeIQx, median (interquartile range)
$26.91( \pm 2.91)$

$25.30( \pm 3.99)$

$96.51( \pm 8.94)$

$81.96( \pm 10.71)$

$$
\begin{gathered}
126(30.5) \\
138(33.4) \\
24(5.8) \\
125(30.3)
\end{gathered}
$$

$140(33.9)$

$191(46.3)$

$82(19.9)$

$11.86( \pm 17.11)$

$$
48 \text { (11.8) }
$$

149 (36.6)

$121(29.7)$

89 (21.9)

39 (9.4)

$24.5( \pm 27.6)$

$77.0( \pm 30.9)$

$71(17.2)$

$22.64(9.83-58.72)$

10.91 (4.56-25.39)

$1.92(0.63-4.31)$

$\begin{aligned} 54.6 & ( \pm 6.24) \\ 520(65.8) & \\ 276(65.9) & \\ 26.74( \pm 3.18) & 0.47 \\ 25.55( \pm 3.89) & 0.57 \\ 96.28( \pm 8.89) & 0.74 \\ 81.74( \pm 11.66) & 0.85\end{aligned}$

249 (31.3)

252 (31.7)

$41(5.2)$

254 (31.9)

0.85

316 (39.7)

364 (45.7)

116 (14.6)

0.02

$9.53( \pm 15.71)$

0.02

84 (10.7)

$300(38.1)$

216 (27.5)

187 (23.8)

0.77

$94(11.8) \quad 0.23$

$20.3( \pm 23.7) \quad 0.01$

$74.7( \pm 29.1) \quad 0.21$

89 (11.2) $\quad 0.01$

${ }^{\text {a }} p$ value assessed by paired $t$ test for continuous variables and $\chi^{2}$ test for categorical variables

b $n$ (number) and \% if not mentioned differently

enzyme activity (AA carriers) associated with a lower CRA risk).

The results of the analysis on interactive effects between genotypes and HCA intake on CRA risk are given in Table 4; we present multivariable adjusted results because age- and sex-adjusted results did not differ materially but were less conservative.
Statistically significant effect modification was found in one gene. SULTIAI predicted phenotypes modified the effect of MeIQx on CRA risk ( $p_{\text {Interaction }}>0.01$ ). The association of MeIQx intake with CRA risk was stronger for slow than for normal predicted phenotypes (4th vs. 1st quartiles OR 3.61, $95 \%$ CI 0.92-14.24, OR 1.22, $95 \%$ CI $0.82-1.80$, respectively). Using MeIQx as a continuous 
Table 3 Odds ratio $\left(95 \%\right.$ CI) for colorectal adenomas by quartiles of heterocyclic aromatic amine intake ${ }^{\mathrm{a}}$, in the case-control study nested in EPIC-Heidelberg

\begin{tabular}{|c|c|c|c|c|}
\hline & No. of cases & No. of controls & $\begin{array}{l}\text { OR }(95 \% \text { CI })^{\mathrm{b}} \\
\text { Unadjusted }\end{array}$ & $\begin{array}{l}\text { OR }(95 \% \text { CI })^{\mathrm{b}} \\
\text { Multiple adjusted }^{\mathrm{c}}\end{array}$ \\
\hline \multicolumn{5}{|l|}{$\mathrm{PhIP}(\mathrm{ng} / \mathrm{d})$} \\
\hline $0-6.72$ & 78 & 199 & 1 & 1 \\
\hline$>6.72-17.62$ & 89 & 199 & $1.15(0.79-1.66)$ & $1.24(0.87-1.78)$ \\
\hline$>17.62-42.31$ & 112 & 199 & $1.47(1.03-2.09)$ & $1.26(0.88-1.81)$ \\
\hline$>42.31$ & 134 & 199 & $1.75(1.23-2.49)$ & $1.81(1.24-2.64)$ \\
\hline$p$ trend & & & 0.002 & 0.006 \\
\hline \multicolumn{5}{|l|}{ MeIQx (ng/d) } \\
\hline $0-3.77$ & 86 & 199 & 1 & 1 \\
\hline$>3.77-9.15$ & 94 & 199 & $1.11(0.78-1.59)$ & $1.11(0.77-1.61)$ \\
\hline$>9.15-18.96$ & 103 & 199 & $1.23(0.86-1.75)$ & $1.20(0.83-1.73)$ \\
\hline$>18.96$ & 130 & 199 & $1.57(1.10-2.24)$ & $1.45(0.99-2.12)$ \\
\hline$p$ trend & & & 0.008 & 0.022 \\
\hline \multicolumn{5}{|l|}{ DiMeIQx (ng/d) } \\
\hline $0-0.58$ & 91 & 199 & 1 & 1 \\
\hline$>0.58-1.69$ & 101 & 199 & $1.11(0.79-1.56)$ & $1.11(0.78-1.57)$ \\
\hline$>1.69-3.70$ & 93 & 199 & $1.02(0.72-1.45)$ & $0.96(0.67-1.37)$ \\
\hline$>3.70$ & 128 & 199 & $1.46(1.01-2.06)$ & $1.35(0.94-1.93)$ \\
\hline$p$ trend & & & 0.027 & 0.045 \\
\hline
\end{tabular}

${ }^{a}$ PhIP:2-amino-1-methyl-6-phenylimidazo[4,5-b]pyridine; MeIQx: 2-amino-3,8-dimethylimidazo[4,5-f]quinoxaline; DiMeIQx: 2-amino-3,4,8dimethylimidazo[4,5-f]quinoxaline

b Conditional logistic regression stratified by case set

c Adjusted for NSAIDs, family history of colorectal cancer, smoking (never, former, current), pack-years of smoking, waist circumference, alcohol intake, fat intake

exposure variable, we observed a statistically significant effect modification $\left(p_{\text {Interaction }}>0.03\right)$ with an increased risk of CRA (OR 7.94, $95 \%$ CI 2.20-28.68) per $50 \mathrm{ng}$ increase in MeIQx for the slow SULTIAl enzyme activities.

For other genes, no significant effect modification was found, but nevertheless we observed significant associations between HCA intake and CRA risk for some phenotypes.

The CYP1A2 normal phenotype and the rapid $C Y P 1 A 2 * 1 F$ (predicted) phenotype did not modify the increasing risk of CRA for higher intake of PhIP, MeIQx, and DiMeIQx ( $\left.p_{\text {Interaction }}>0.05\right)$. However, the association of PhIP intake with CRA was significant only in the rapid phenotype for the 4th quartile compared with the 1st quartile (OR 1.77; $95 \%$ CI 1.05-2.98). For NAT1 genotypes, there was a significantly increased risk of CRA in the $* 4 / * 4$ alleles group (wild type) for the 4 th quartile of PhIP compared with the 1st quartile (OR 1.91; $95 \%$ CI 1.14-3.21), but the interaction between (predicted) phenotype and HCA intake on CRA risk was not statistically significant $\left(p_{\text {Interaction }}>0.05\right)$. For NAT2 slow metabolizing phenotype, we observed a significantly increased CRA risk for high vs. low intake of PhIP (OR 1.80, $95 \%$ CI
1.14-3.21). Intake of PhIP with CRA risk for the SULTIAI normal phenotype (OR 1.61, $95 \%$ CI 1.08-2.40) showed a statistically significantly positive association. An increased risk of CRA was observed with increasing PhIP intake for the GSTA1 normal predicted phenotype in the 4th quartile compared to the 1st quartile (OR 1.82, $95 \%$ CI 1.20-2.77). In contrast, the association of MeIQx and DiMeIQx with the risk of CRA did not increase significantly for the GSTA1 phenotype. UGTIA7 phenotypes had no modifying effect on the association of HCA with CRA. However, the increase in CRA risk with increasing HCA intake was strongest among participants with slow phenotype (OR $3.02,95 \%$ CI 1.02-8.94, 1st vs. 4th quartile). For the rapid phenotype of UGTIA9, there was a statistically significant increased risk of CRA with an OR of 1.97 (95\% CI 1.23-3.16) in the 4th quartile of PhIP compared to the 1st quartile, but there was no significant association for the normal activity phenotype. All analyses were repeated using HCA intake as continuous variable.

Figure 1 shows the associations of HCA intake with CRA risk for three phenotype groups derived from genetic variants in HCA-metabolizing enzymes. Increasing HCA intake was positively associated with CRA for the low-risk groups. For PhIP, this result was statistically significant 
Table 4 OR (95\% CI) for the association between the intake of PhIP, MeIQx, and DiMeIQx stratified in quartiles with the risk of CRA in strata of genetic polymorphisms in the EPIC-Heidelberg nested case-control study $(n=1,209)$

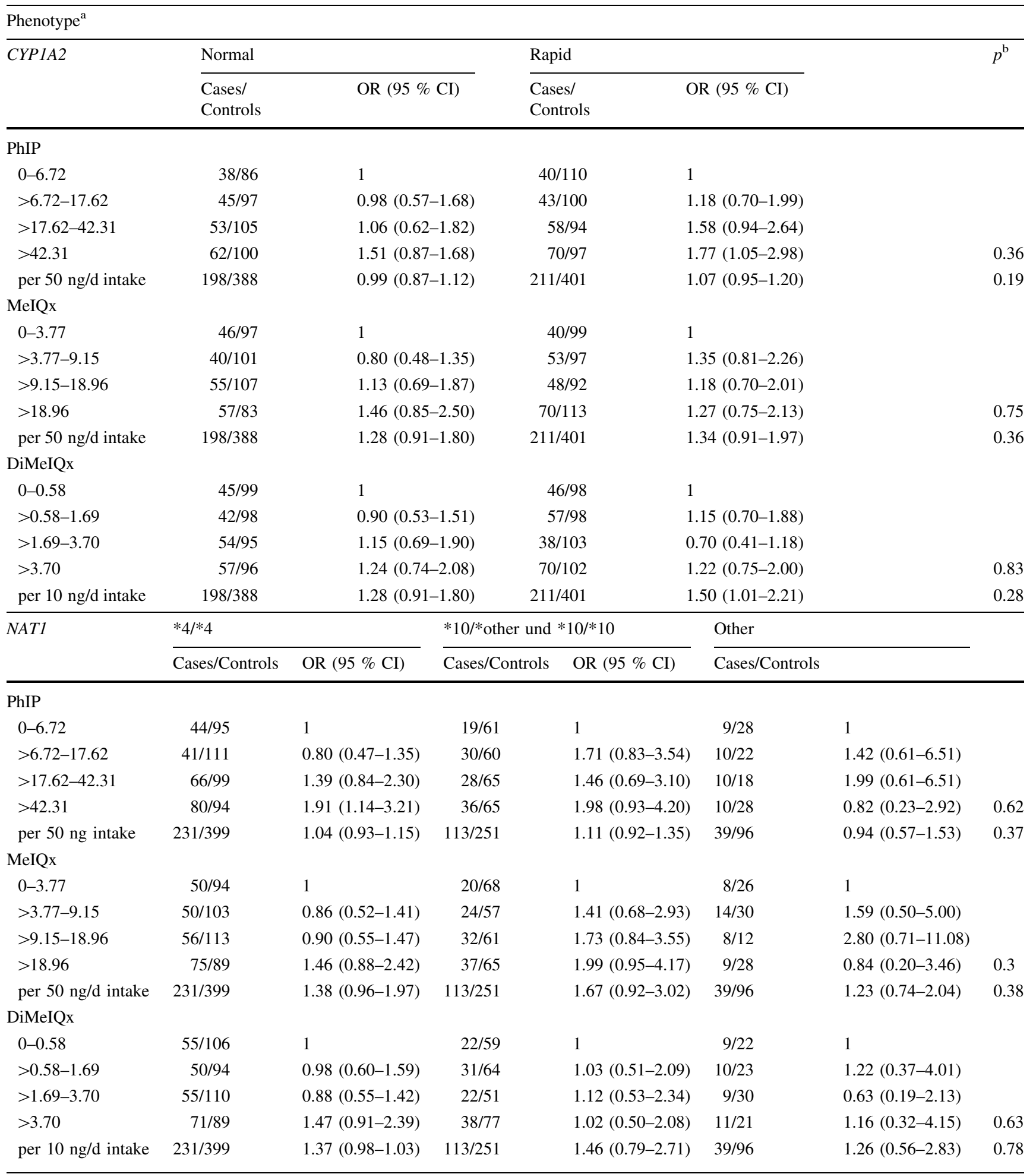


Table 4 continued

\begin{tabular}{|c|c|c|c|c|c|}
\hline \multirow[t]{2}{*}{ NAT2 } & \multicolumn{2}{|l|}{ Normal } & \multicolumn{2}{|l|}{ Slow } & \\
\hline & Cases/Controls & OR $(95 \%$ CI $)$ & Cases/Controls & OR $(95 \% \mathrm{CI})$ & \\
\hline \multicolumn{6}{|l|}{$\mathrm{PhIP}$} \\
\hline $0-6.72$ & $30 / 89$ & 1 & $47 / 98$ & 1 & \\
\hline$>6.72-17.62$ & $42 / 86$ & $1.43(0.80-2.55)$ & $43 / 106$ & $0.85(0.51-1.42)$ & \\
\hline$>17.62-42.31$ & $34 / 86$ & $1.11(0.60-2.04)$ & $73 / 107$ & $1.38(0.85-2.24)$ & \\
\hline$>42.31$ & $52 / 90$ & $1.68(0.93-3.05)$ & $76 / 97$ & $1.80(1.09-2.99)$ & 0.8 \\
\hline per $50 \mathrm{ng} / \mathrm{d}$ intake & $158 / 351$ & $1.02(0.88-1.87)$ & $239 / 408$ & $1.05(0.95-1.17)$ & 0.81 \\
\hline \multicolumn{6}{|l|}{ MeIQx } \\
\hline $0-3.77$ & $31 / 87$ & 1 & $52 / 102$ & 1 & \\
\hline$>3.77-9.15$ & $40 / 91$ & $1.39(0.78-2.47)$ & $52 / 103$ & $0.89(0.55-1.46)$ & \\
\hline$>9.15-18.96$ & $34 / 89$ & $1.15(0.63-2.09)$ & $65 / 103$ & $1.18(0.73-1.89)$ & \\
\hline$>18.96$ & $53 / 84$ & $1.87(1.02-3.43)$ & $70 / 100$ & $1.27(0.78-2.08)$ & 0.32 \\
\hline per $50 \mathrm{ng} / \mathrm{d}$ intake & $158 / 351$ & $1.54(1.00-2.37)$ & $239 / 408$ & $1.27(0.91-1.77)$ & 0.5 \\
\hline \multicolumn{6}{|l|}{ DiMeIQx } \\
\hline $0-0.58$ & $30 / 84$ & 1 & $58 / 109$ & 1 & \\
\hline$>0.58-1.69$ & $42 / 83$ & $1.42(0.79-2.53)$ & $55 / 100$ & $0.99(0.62-1.59)$ & \\
\hline$>1.69-3.70$ & $33 / 88$ & $0.97(0.53-1.75)$ & $58 / 104$ & $0.94(0.58-1.50)$ & \\
\hline$>3.70$ & $53 / 96$ & $1.51(0.85-2.69)$ & $68 / 95$ & $1.29(0.80-2.06)$ & 0.63 \\
\hline per $10 \mathrm{ng} / \mathrm{d}$ intake & $158 / 351$ & $0.13(0.00-7.23)$ & $239 / 408$ & $1.72(1.12-2.64)$ & 0.49 \\
\hline \multirow[t]{2}{*}{ SULT1A1 } & \multicolumn{2}{|l|}{ Normal } & \multicolumn{2}{|l|}{ Slow } & \\
\hline & Cases/Controls & OR $(95 \%$ CI $)$ & Cases/Controls & OR $(95 \% \mathrm{CI})$ & \\
\hline \multicolumn{6}{|l|}{$\mathrm{PhIP}$} \\
\hline $0-6.72$ & $67 / 175$ & 1 & $11 / 19$ & 1 & \\
\hline$>6.72-17.62$ & $84 / 179$ & $1.23(0.83-1.81)$ & $4 / 18$ & $0.35(0.08-1.53)$ & \\
\hline$>17.62-42.31$ & $100 / 180$ & $1.37(0.93-2.02)$ & $11 / 19$ & $0.98(0.29-3.32)$ & \\
\hline$>42.31$ & $113 / 178$ & $1.61(1.08-2.40)$ & $19 / 19$ & $2.07(0.63-6.80)$ & 0.28 \\
\hline per $50 \mathrm{ng}$ intake & $364 / 712$ & $1.01(0.93-1.11)$ & $45 / 75$ & $1.36(0.92-2.02)$ & 0.12 \\
\hline \multicolumn{6}{|l|}{ MeIQx } \\
\hline $0-3.77$ & $76 / 178$ & 1 & $10 / 16$ & 1 & \\
\hline$>3.77-9.15$ & $88 / 174$ & $1.18(0.81-1.72)$ & $5 / 24$ & $0.25(0.06-1.10)$ & \\
\hline$>9.15-18.96$ & $94 / 177$ & $1.21(0.83-1.77)$ & $9 / 22$ & $0.70(0.20-2.43)$ & \\
\hline$>18.96$ & $106 / 183$ & $1.22(0.82-1.80)$ & $21 / 13$ & $3.61(0.92-14.24)$ & 0.01 \\
\hline per $50 \mathrm{ng} / \mathrm{d}$ intake & $364 / 712$ & $1.19(0.93-1.53)$ & $45 / 75$ & $7.94(2.20-28.68)$ & 0.03 \\
\hline \multicolumn{6}{|l|}{ DiMeIQx } \\
\hline $0-0.58$ & $83 / 181$ & 1 & $8 / 16$ & 1 & \\
\hline$>0.58-1.69$ & $87 / 175$ & $1.07(0.74-1.55)$ & $12 / 19$ & $0.89(0.25-3.14)$ & \\
\hline$>1.69-3.70$ & $84 / 170$ & $1.01(0.69-1.47)$ & $8 / 28$ & $0.50(0.13-1.82)$ & \\
\hline$>3.70$ & $110 / 186$ & $1.17(0.80-1.69)$ & $17 / 12$ & $2.94(0.80-10.77)$ & 0.08 \\
\hline per $10 \mathrm{ng} / \mathrm{d}$ intake & $364 / 712$ & $1.26(0.96-1.65$ & $45 / 75$ & $4.07(1.25-13.26)$ & 0.07 \\
\hline \multirow[t]{2}{*}{ GSTA1 } & \multicolumn{2}{|l|}{ Normal } & \multicolumn{2}{|l|}{ Slow } & \\
\hline & Cases/Controls & OR $(95 \%$ CI $)$ & Cases/Controls & OR (95 \% CI) & \\
\hline \multicolumn{6}{|l|}{$\mathrm{PhIP}$} \\
\hline $0-6.72$ & $66 / 163$ & 1 & $12 / 36$ & 1 & \\
\hline$>6.72-17.62$ & $78 / 150$ & $1.29(0.86-1.94)$ & $11 / 47$ & $0.73(0.28-1.93)$ & \\
\hline$>17.62-42.31$ & $94 / 165$ & $1.33(0.89-1.98)$ & $18 / 34$ & $1.69(0.66-4.34)$ & \\
\hline
\end{tabular}


Table 4 continued

\begin{tabular}{|c|c|c|c|c|c|c|c|c|c|c|}
\hline \multirow[t]{2}{*}{ GSTA1 } & \multicolumn{4}{|l|}{ Normal } & \multicolumn{4}{|c|}{ Slow } & & \\
\hline & \multicolumn{2}{|l|}{$\begin{array}{l}\text { Cases/ } \\
\text { Controls }\end{array}$} & \multicolumn{2}{|c|}{ OR $(95 \% \mathrm{CI})$} & \multicolumn{2}{|c|}{$\begin{array}{l}\text { Cases/ } \\
\text { Controls }\end{array}$} & \multicolumn{3}{|c|}{ OR $(95 \% \mathrm{CI})$} & \\
\hline$>42.31$ & \multicolumn{2}{|l|}{$111 / 145$} & \multicolumn{2}{|c|}{$1.82(1.20-2.77)$} & \multicolumn{2}{|c|}{$23 / 54$} & \multicolumn{2}{|c|}{$1.51(0.61-3.77)$} & & 0.43 \\
\hline $\begin{array}{l}\text { per } 50 \mathrm{ng} / \mathrm{d} \\
\text { intake }\end{array}$ & \multicolumn{2}{|l|}{$349 / 623$} & \multicolumn{2}{|c|}{$1.04(0.95-1.15)$} & \multicolumn{2}{|c|}{$64 / 171$} & \multicolumn{2}{|c|}{$1.03(0.86-1.25)$} & & 0.6 \\
\hline \multicolumn{11}{|l|}{ MeIQx } \\
\hline $0-3.77$ & \multicolumn{2}{|l|}{$72 / 154$} & \multicolumn{2}{|l|}{1} & \multicolumn{2}{|c|}{$14 / 44$} & \multicolumn{2}{|l|}{1} & & \\
\hline$>3.77-9.15$ & \multicolumn{2}{|l|}{$78 / 156$} & \multicolumn{2}{|c|}{$1.07(0.72-1.60)$} & $16 /$ & & 1.1 & $(0.50-2.79)$ & & \\
\hline$>9.15-18.96$ & $91 / 158$ & & $1.23(0.82$ & $1.82)$ & $12 /$ & & 0.7 & $(0.28-1.86)$ & & \\
\hline$>18.96$ & $108 / 155$ & & $1.34(0.89$ & $1.82)$ & $22 /$ & & 1.4 & $(0.62-3.47)$ & & 0.91 \\
\hline $\begin{array}{l}\text { per } 50 \mathrm{ng} / \mathrm{d} \\
\text { intake }\end{array}$ & $349 / 623$ & & $1.44(1.05$ & 1.97) & $64 / 1^{-}$ & & 0.9 & $(0.55-1.80)$ & & 0.14 \\
\hline DiMeIQx & & & & & & & & & & \\
\hline $0-0.58$ & $76 / 149$ & & 1 & & $15 /$ & & 1 & & & \\
\hline$>0.58-1.69$ & $87 / 165$ & & $1.00(0.68$ & $1.47)$ & $14 /$ & & 1.4 & $(0.58-3.46)$ & & \\
\hline$>1.69-3.70$ & $81 / 152$ & & $0.98(0.66$ & $1.45)$ & $12 /$ & & 0.6 & $(0.26-1.63)$ & & \\
\hline$>3.70$ & $105 / 157$ & & $1.15(0.78$ & $1.71)$ & $23 /$ & & 1.7 & $(0.75-4.08)$ & & 0.62 \\
\hline $\begin{array}{l}\text { per } 10 \mathrm{ng} / \mathrm{d} \\
\text { intake }\end{array}$ & $349 / 623$ & & $1.62(1.17$ & $2.24)$ & $64 / 1$ & & 1.0 & $(0.62-1.67)$ & & 0.08 \\
\hline UGT1A7 & Intermediate & & & Rapid & & & & Slow & & \\
\hline & Cases/Controls & & $5 \% \mathrm{CI})$ & Cases/Co & rols & OR $(95 \%$ & & Cases/Controls & OR $(95 \% \mathrm{CI})$ & \\
\hline $\mathrm{PhIP}$ & & & & & & & & & & \\
\hline $0-6.72$ & $38 / 84$ & 1 & & $31 / 80$ & & 1 & & $9 / 33$ & 1 & \\
\hline$>6.72-17.62$ & $35 / 95$ & & $0.45-1.37)$ & $35 / 76$ & & $1.29(0.70$ & & $18 / 25$ & $2.77(0.99-7.77)$ & \\
\hline$>17.62-42.31$ & $63 / 102$ & & $0.73-2.06)$ & $32 / 67$ & & $1.24(0.66$ & & $16 / 30$ & $2.20(0.77-6.34)$ & \\
\hline$>42.31$ & $65 / 95$ & & $0.80-2.33)$ & $49 / 80$ & & $1.71(0.91-$ & & $19 / 22$ & $3.02(1.02-8.94)$ & 0.94 \\
\hline per $50 \mathrm{ng} / \mathrm{d}$ intake & $201 / 376$ & & $0.86-1.13)$ & $147 / 303$ & & $1.03(0.90$ & & $62 / 110$ & $1.16(0.92-1.47)$ & 0.57 \\
\hline MeIQx & & & & & & & & & & \\
\hline $0-3.77$ & $41 / 89$ & 1 & & $33 / 72$ & & 1 & & $12 / 34$ & 1 & \\
\hline$>3.77-9.15$ & $43 / 93$ & & $0.57-1.65)$ & $35 / 78$ & & $1.06(0.58$ & & $15 / 28$ & $1.59(0.60-4.24)$ & \\
\hline$>9.15-18.96$ & $53 / 102$ & & $0.63-1.76)$ & $35 / 74$ & & $1.05(0.57$ & & $15 / 23$ & $2.04(0.74-5.86)$ & \\
\hline$>18.96$ & $64 / 92$ & & $0.76-2.17)$ & $44 / 79$ & & $1.23(0.66$ & & $20 / 25$ & $2.09(0.75-5.85)$ & 0.93 \\
\hline per $50 \mathrm{ng} / \mathrm{d}$ intake & $201 / 376$ & & $0.87-1.60)$ & $147 / 303$ & & $1.46(0.91-$ & & $62 / 110$ & $1.24(0.54-2.86)$ & 0.79 \\
\hline DiMeIQx & & & & & & & & & & \\
\hline $0-0.58$ & $46 / 86$ & 1 & & $26 / 72$ & & 1 & & $19 / 38$ & 1 & \\
\hline$>0.58-1.69$ & $41 / 94$ & & $0.45-1.27)$ & $46 / 76$ & & $1.64(0.89$ & & $13 / 26$ & $1.07(0.42-2.71)$ & \\
\hline$>1.69-3.70$ & $47 / 101$ & & $0.49-1.35)$ & $36 / 79$ & & $1.15(0.61-$ & & $10 / 19$ & $1.09(0.40-2.97)$ & \\
\hline$>3.70$ & $67 / 95$ & & $0.70-1.91)$ & $39 / 76$ & & $1.36(0.72$ & & $20 / 27$ & $1.31(0.52-3.33)$ & 0.56 \\
\hline per $10 \mathrm{ng} / \mathrm{d}$ intake & $201 / 376$ & & $0.89-1.74)$ & $147 / 303$ & & $1.41(0.86$ & 29) & $62 / 110$ & $1.53(0.68-3.40)$ & 0.73 \\
\hline UGT1A9 & Normal & & & & $\mathrm{Ra}$ & & & & & \\
\hline & Cases/Contı & & OR $(95$ & CI) & $\mathrm{Ca}$ & s/Controls & & $\mathrm{R}(95 \% \mathrm{CI})$ & & \\
\hline PhIP & & & & & & & & & & \\
\hline $0-6.72$ & $31 / 72$ & & 1 & & & 127 & 1 & & & \\
\hline$>6.72-17.62$ & $37 / 76$ & & $1.05(0.5$ & $-1.91)$ & & 121 & & $24(0.77-2.01)$ & & \\
\hline$>17.62-42.31$ & $44 / 73$ & & $1.37(0.7$ & $-2.53)$ & & 126 & & $43(0.90-2.29)$ & & \\
\hline$>42.31$ & $40 / 73$ & & $1.28(0.6$ & $-2.53)$ & & 125 & & $97(1.23-3.16)$ & & 0.12 \\
\hline per $50 \mathrm{ng} / \mathrm{d}$ intake & $152 / 294$ & & $1.01(0.8$ & $-1.18)$ & 25 & 495 & & 05 (0.94-1.16) & & 0.52 \\
\hline
\end{tabular}


Table 4 continued

\begin{tabular}{|c|c|c|c|c|c|}
\hline \multirow[t]{2}{*}{ UGT1A9 } & \multicolumn{2}{|l|}{ Normal } & \multicolumn{2}{|l|}{ Rapid } & \\
\hline & $\begin{array}{l}\text { Cases/ } \\
\text { Controls }\end{array}$ & OR $(95 \% \mathrm{CI})$ & $\begin{array}{l}\text { Cases/ } \\
\text { Controls }\end{array}$ & OR $(95 \% \mathrm{CI})$ & \\
\hline \multicolumn{6}{|l|}{ MeIQx } \\
\hline $0-3.77$ & $35 / 70$ & 1 & $51 / 128$ & 1 & \\
\hline$>3.77-9.15$ & $37 / 79$ & $0.94(0.52-1.68)$ & $56 / 120$ & $1.25(0.78-1.99)$ & \\
\hline$>9.15-18.96$ & $37 / 77$ & $1.04(0.57-1.88)$ & $66 / 122$ & $1.32(0.83-2.09)$ & \\
\hline$>18.96$ & $43 / 68$ & $1.27(0.67-2.41)$ & $86 / 129$ & $1.55(0.98-2.46)$ & 0.47 \\
\hline $\begin{array}{l}\text { per } 50 \mathrm{ng} / \mathrm{d} \\
\text { intake }\end{array}$ & $152 / 294$ & $1.05(0.95-1.16)$ & $259 / 495$ & $1.06(0.99-1.03)$ & 0.55 \\
\hline \multicolumn{6}{|l|}{ DiMeIQx } \\
\hline $0-0.58$ & $41 / 80$ & 1 & $50 / 117$ & 1 & \\
\hline$>0.58-1.69$ & $34 / 69$ & $1.06(0.59-1.88)$ & $66 / 129$ & $1.10(0.70-1.74)$ & \\
\hline$>1.69-3.70$ & $32 / 62$ & $1.07(0.59-1.93)$ & $61 / 137$ & $0.94(0.59-1.49)$ & \\
\hline$>3.70$ & $45 / 83$ & $1.07(0.60-1.90)$ & $82 / 116$ & $1.50(0.94-2.37)$ & 0.16 \\
\hline $\begin{array}{l}\text { per } 10 \mathrm{ng} / \mathrm{d} \\
\text { intake }\end{array}$ & $152 / 294$ & $1.52(0.95-2.44)$ & $259 / 495$ & $1.34(0.98-1.83)$ & 0.99 \\
\hline
\end{tabular}

${ }^{a}$ All results are multivariable adjusted

${ }^{\mathrm{b}} p$ value for test for interaction between HCA intake and phenotype

(4th vs. 1st quartile, OR 2.25, $95 \%$ CI 1.33-3.83). Between phenotype risk groups and HCA intake, no significant interaction $\left(p_{\text {Interaction }}>0.05\right)$ was observed.

\section{Discussion}

In the current study, we observed a statistically significant increased risk of CRA with increasing HCA intake (PhIP, MeIQx, and DiMeIQx), as shown previously in a cohort design [21]. In general, HCA intake and CRA risk were positively associated when stratified by phenotypes of single enzymes. There was no effect modification on the association between HCA intake and CRA risk by genetic polymorphisms, with the exception of SULT1A1 and MeIQx intake. Comparing groups of enzymes, we did not find any significant effect modification. However, in the 4th quartile of slow HCA-activating phenotypes or of rapid HCA-detoxifying phenotypes, significant results were obtained for PhIP.

CYP1A2 phase 1 enzymes are involved in the first step of metabolism of HCA. The $C Y P 1 A 2 * \mathrm{~F}$ polymorphism is associated with enhanced enzyme activity [26]. Nevertheless, in our study, there was no modifying effect of CYP1A2 on the association of HCA with CRA risk, which is in accordance with other studies looking at meat and HCA intake on the CRC or CRA risk [13, 19].

There are still uncertainties about definition of phenotypes predicted from genotypes of $N A T 1$, due to the lack of comprehension of NAT1 effects [40]. This might be a reason for inconsistent results. The study of Ishibe et al. [13] with 146 cases observed an increased CRA risk for NAT1 rapid acetylators with high MeIQx consumption. The rapid acetylators were defined as having at least one $N A T 1 * 10$ allele. In contrast, the study of Shin et al. [19] with 557 CRA cases considered $N A T 1 * 10$ and $N A T 1 * 11$ alleles as rapid acetylators only in combination or heterozygous with a $N A T 1 * 3$ and $N A T 1 * 4$ allele. They observed an association of high HCA intake with risk for polyps among participants with high activity NAT1 or NAT2 genotypes. Similar results were obtained from the abovementioned Hawaiian study [41]. The study of Tiemersma et al. [14] looked at total meat intake and found a higher CRA risk for slow NAT2 acetylators than for intermediate/ fast phenotypes. In our study, there was no effect modification by NAT1 or NAT2. Concerning main effects, we had previously reported that carriers of the combined NAT2 alleles encoding for enzymes with medium (versus slow) activity had a significantly lower adenoma risk $(\mathrm{OR}=0.75 ; 95 \% \mathrm{CI}$ 0.85-0.97). Compared to individuals carrying two $N A T 1 * 4$ alleles, all other predicted NAT1 phenotypes were associated with a non-significantly decreased risk of colorectal adenomas [27].

We observed modifying effects of SULT1A1 phenotypes on the association of MeIQx intake with CRA risk, with slow phenotypes having the highest CRA risk. Up to our knowledge, there is one Dutch study [14] examining the effect of SULTIAI polymorphisms on the association of meat consumption with CRA risk in a case-control setting with 431 CRA cases. It did not reveal any effect 
Fig. 1 Multivariate adjusted OR of CRA according to quartiles of PhIP (a), MeIQx (b), and DiMeIQx (c) intake and three phenotype groups derived from genetic variants in HCA-metabolizing enzymes. Low-CRA-risk group is defined when at least four of seven following phenotypes were available: rapid CYP1A2, NAT1*10/*10, or NAT1*10/other, rapid NAT2, intermediate SULT1A1, slow GSTA1, slow UGT1A7, intermediate UGT1A9. High-CRA-risk group is defined when at least four of seven following phenotypes were available: intermediate CYP1A2, NAT1*4/*4, slow NAT2, slow SULT1A1, intermediate GSTA1, rapid UGT1A7, rapid UGT1A9. The remaining enzyme combinations were assigned to the intermediate-CRA-risk group. The reference categories consist of participants in the lowest quartile of HCAs. T-bars indicate $95 \%$ confidence intervals for the ORs of CRA risk. $p_{\text {Interaction }}$ was $>0.05$ between phenotype risk groups and HCA intake. Cases/controls were distributed as follows for PhIP (1st to 4th HCA quartiles): 11/36, 17/26, 19/38, 30/27 (low-CRA-risk group); 65/157, 63/164, 89/154, 101/161 (intermediate-CRA-risk group); 2/6, 9/9, 4/7, 3/11 (highCRA-risk group). For MeIQx (1st to 4th HCA quartiles): 15/34, 14/26, 23/34, 25, 33 (low-CRA-risk group); 66/156, 74/165, 76/162, 102/153 (intermediate-CRA-risk group); 5/9, 6/8, 4/3, 3/13 (highCRA-risk group). For DiMeIQx (1st to 4th HCA quartiles): 14/34, 23/34, 19/34, 21/25 (low-CRA-risk group); 70/156, 74/160, 71/161, 103/159 (intermediate-CRA-risk group); 7/9, 4/5, 3/4, 4/15 (highCRA-risk group)

modification of SULTIA1, although intermediate/fast phenotypes had a higher CRA risk than slow phenotypes. Concerning CRC, a Canadian case-control study [42] found a modifying effect of SULTIAI combined with $C Y P 1 B 1$ on the association between red meat doneness intake and CRC risk. The SULTIAl GG genotype that corresponds to our intermediate phenotype had higher CRC risk than the GA/AA genotypes. A German case-control study [43] reported that low-activity SULTIAI*2 alleles were associated with higher CRC risk. Since SULTs are involved in both activation and detoxification of HCAs [44], this may explain the inconsistent results. Due to multiple comparisons and the small number of our slow SULT1A1 phenotypes, it warrants further study on the effects of SULT enzymes on HCA intake and CRA risk.

According to the metabolizing process of HCAs, enzymes of the GST and UGT family detoxify carcinogenic HCAs. Thus, slow detoxifying polymorphisms of this group may be associated with a higher CRA risk than high detoxifying GST and UGT enzymes. However, we had previously reported a decreased risk of colorectal adenomas among those participants with the low-activity GSTA1 phenotype [27], which has also been reported in a study on gastric cancer [45]. When looking at interaction effects, we observed a significant association of PhIP intake with CRA risk for the GSTA1 phenotype with normal activity. However, one case-control study [46] looked at consumption of well-done meat and CRC and found a higher OR for subjects with the combination of high intake of well-done meat and the low-activity GSTAl phenotype.

One case-control study examined the effect of UGTIA7 [36] and observed an increased CRC risk for PhIP and
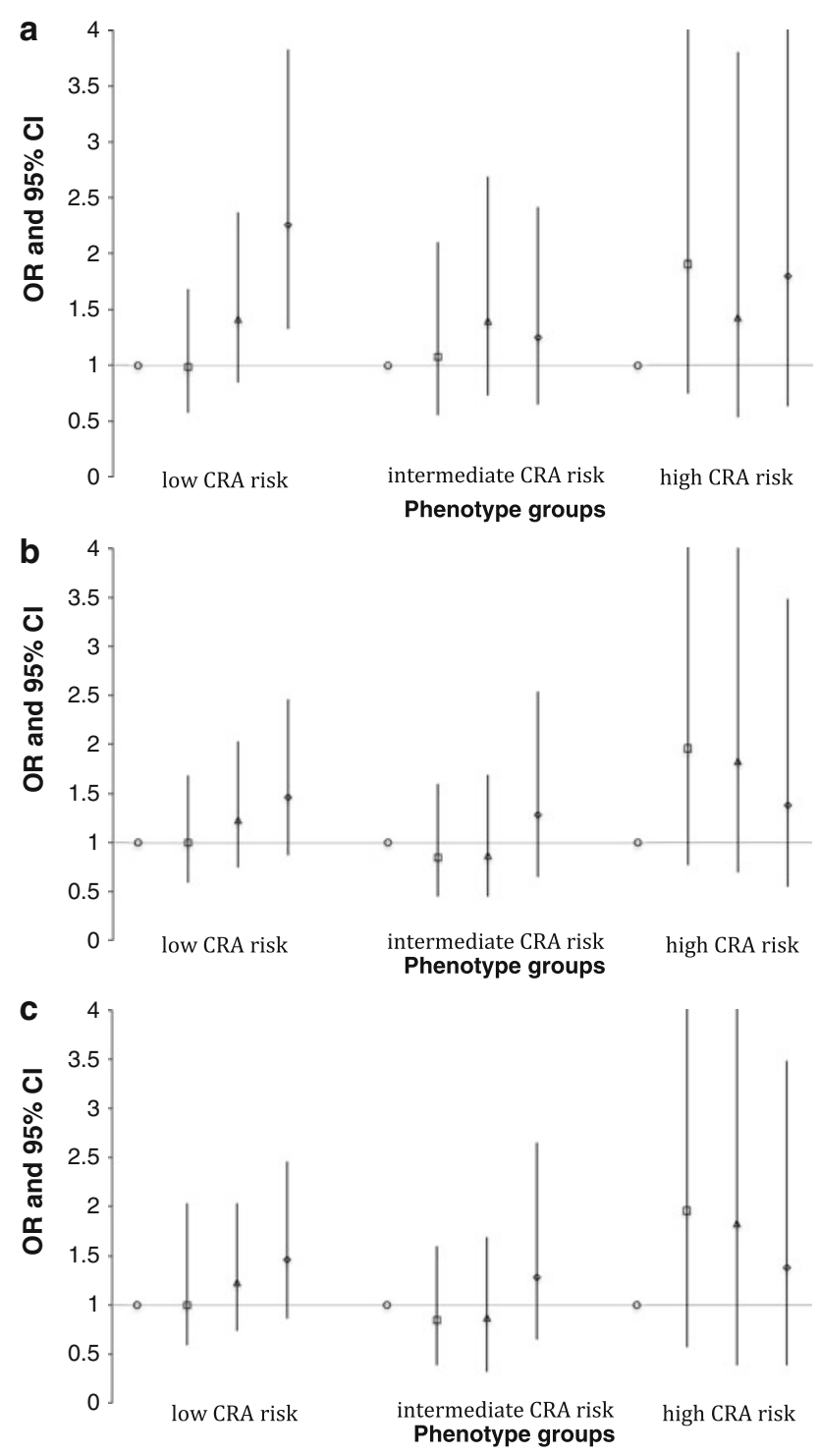

DiMeIQx regardless of phenotypes (slow vs. high/intermediate phenotypes). We observed no effect modification by UGTIA7 phenotype, but observed a stronger association between PhIP intake and CRA risk among those with slow phenotype. Concerning UGT1A9, we also observed no statistically significant effect modification, although there was once again a stronger association between PhIP intake and CRA risk among those with slow phenotype. To the best of our knowledge, no other studies concerning this gene have been published in this regard.

To the best of our knowledge, there is no study comparing a score of high-CRA-risk phenotypes vs. low-CRArisk phenotypes constructed on the basis of the genotype information. Although the test for interaction does not indicate an overall effect modification, we got indication of a dose-response relationship in subjects with a "low CRA risk" phenotype (significant effect for the highest versus 
lowest intake of $\mathrm{PhIP}$ ). Since some enzymes are involved in both activation and detoxification of HCAs, there may be a misclassification of these enzymes in high-, intermediate-, or low-risk phenotype groups. Moreover, there were no participants in our study who had the maximum number of exact phenotype combination to be assigned in the highor low-risk group. Therefore, we had to specify how much of the predicted phenotypes had to be in each group to be counted as high- or low-risk phenotype group, which implicates some inaccuracy. The lack of findings does, thus, not suggest that SNPs have no influence on the association of HCA with CRA but might be due to a lesssensitive definition of high- and low-risk groups.

A major strength of the study is its prospective design. Given that this was a nested case-control study, the selection bias was minimal. Further strengths of the study included the well-characterized study population with comparable cases and controls. All cases had medically confirmed diagnoses of adenomas and controls were free of adenomas (at least confirmed once by colonoscopy). The detailed assessments of HCA intake and the allelic characterization of chosen genes involved in the HCA metabolism are additional advantages of our study. Furthermore, we were able to adjust for known confounders in our analyses, but residual confounding cannot be completely excluded.

Limitations of this study are the possible misclassification of HCA intake when using a photograph-based questionnaire. This choice of using questionnaire is nevertheless justified, because the more accurate estimation of HCA in biomaterials, for example, in hair [47], is very expensive. Hence, this method is not available for large epidemiological studies up to date. The potential lack of power in our study, in particular with respect to the CRArisk groups, is a limitation that should be overcome with larger studies. Additional limitation is the inclusion of controls with negative colonoscopy, which might have biased the results. These participants had the colonoscopy before study inclusion and so, being in need for a colonoscopy, they might be less healthy and/or more health conscious.

Further limitation is the potential for false-positive results based on multiple comparisons. Therefore, the significant effect modification of SULTIAI may be due to chance. Finally, HCA intake has been assessed in the second follow-up, and some cases had been diagnosed before this date. Excluding these cases from our analysis did not materially change the observed associations.

HCA enzyme activity can be influenced not only by genes but also by environmental factors, such as alcohol consumption, smoking, and diet [48-50]. In addition to HCAs, other genotoxic agents such as nitrosamines and polycyclic aromatic hydrocarbons, which develop during meat cooking or meat processing, may increase the CRA and CRC risk [14, 16, 41, 50]. Up to date it remains rather unclear in which relationship different genotoxic agents interact to increase the CRA risk. In addition, we may have missed some other possible phenotypes that modify the association between HCA and CRA (e.g., GSTM1). Genegene interactions may also play a role in the genesis of CRA depending on HCA intake.

In summary, in this study, HCA intake and CRA risk were positively associated, independent of polymorphisms in genes involved. Because of the complex associations of environmental factors, genotoxic agents and genetic variants, modifying effects of genotypes and their functional correlates (enzyme activities, here called phenotypes) might be underestimated. They are not sufficiently illuminated up to date and need further research.

Acknowledgments This study was granted by the Kurt-EberhardBode Foundation.

Conflict of interest The authors declare that they have no conflict of interest.

\section{References}

1. Boyle P, Ferlay J (2005) Cancer incidence and mortality in Europe, 2004. Ann Oncol 16(3):481-488. doi:10.1093/annonc/ mdi098

2. Chan DS, Lau R, Aune D, Vieira R, Greenwood DC, Kampman E, Norat T (2011) Red and processed meat and colorectal cancer incidence: meta-analysis of prospective studies. PLoS One 6(6):e20456. doi:10.1371/journal.pone.0020456

3. Sinha R (2002) An epidemiologic approach to studying heterocyclic amines. Mutat Res 506-507:197-204

4. Sinha R, Norat T (2002) Meat cooking and cancer risk. IARC Sci Publ 156:181-186

5. Murkovic M (2004) Formation of heterocyclic aromatic amines in model systems. J Chromatogr B Analyt Technol Biomed Life Sci 802(1):3-10. doi:10.1016/j.jchromb.2003.09.026

6. Strickland PT, Qian Z, Friesen MD, Rothman N, Sinha R (2002) Metabolites of 2-amino-1-methyl-6-phenylimidazo(4,5-b)pyridine (PhIP) in human urine after consumption of charbroiled or fried beef. Mutat Res 506-507:163-173

7. Skog KI, Johansson MA, Jagerstad MI (1998) Carcinogenic heterocyclic amines in model systems and cooked foods: a review on formation, occurrence and intake. Food Chem Toxicol 36(9-10):879-896

8. Zheng W, Lee SA (2009) Well-done meat intake, heterocyclic amine exposure, and cancer risk. Nutr Cancer 61(4):437-446. doi:10.1080/01635580802710741

9. Shirai T, Tamano S, Sano M, Masui T, Hasegawa R, Ito N (1995) Carcinogenicity of 2-amino-1-methyl-6-phenylimidazo[4,5b]pyridine (PhIP) in rats: dose-response studies. Princess Takamatsu Symp 23:232-239

10. Adamson RH, Snyderwine EG, Thorgeirsson UP, Schut HA, Turesky RJ, Thorgeirsson SS, Takayama S, Sugimura T (1990) Metabolic processing and carcinogenicity of heterocyclic amines in nonhuman primates. Princess Takamatsu Symp 21:289-301

11. Risio M (2010) The natural history of adenomas. Best Pract Res Clin Gastroenterol 24(3):271-280. doi:10.1016/j.bpg.2010. 04.005 
12. Sinha R, Kulldorff M, Chow WH, Denobile J, Rothman N (2001) Dietary intake of heterocyclic amines, meat-derived mutagenic activity, and risk of colorectal adenomas. Cancer Epidemiol Biomarkers Prev 10(5):559-562

13. Ishibe N, Sinha R, Hein DW, Kulldorff M, Strickland P, Fretland AJ, Chow WH, Kadlubar FF, Lang NP, Rothman N (2002) Genetic polymorphisms in heterocyclic amine metabolism and risk of colorectal adenomas. Pharmacogenetics 12(2):145-150

14. Tiemersma EW, Voskuil DW, Bunschoten A, Hogendoorn EA, Witteman BJ, Nagengast FM, Glatt H, Kok FJ, Kampman E (2004) Risk of colorectal adenomas in relation to meat consumption, meat preparation, and genetic susceptibility in a Dutch population. Cancer Causes Control 15(3):225-236. doi:10.1023 /B:CACO.0000024263.44973.92

15. Gunter MJ, Probst-Hensch NM, Cortessis VK, Kulldorff M, Haile RW, Sinha R (2005) Meat intake, cooking-related mutagens and risk of colorectal adenoma in a sigmoidoscopy-based case-control study. Carcinogenesis 26(3):637-642. doi:10.1093/carcin/bgh350

16. Sinha R, Peters U, Cross AJ, Kulldorff M, Weissfeld JL, Pinsky PF, Rothman N, Hayes RB (2005) Meat, meat cooking methods and preservation, and risk for colorectal adenoma. Cancer Res 65(17):8034-8041. doi:10.1158/0008-5472.CAN-04-3429

17. Wu K, Giovannucci E, Byrne C, Platz EA, Fuchs C, Willett WC, Sinha R (2006) Meat mutagens and risk of distal colon adenoma in a cohort of U.S. men. Cancer Epidemiol Biomarkers Prev 15(6):1120-1125. doi:10.1158/1055-9965.EPI-05-0782

18. Shin A, Shrubsole MJ, Ness RM, Wu H, Sinha R, Smalley WE, Shyr Y, Zheng W (2007) Meat and meat-mutagen intake, doneness preference and the risk of colorectal polyps: the Tennessee Colorectal Polyp Study. Int J Cancer 121(1):136-142. doi: 10.1002/ijc. 22664

19. Shin A, Shrubsole MJ, Rice JM, Cai Q, Doll MA, Long J, Smalley WE, Shyr Y, Sinha R, Ness RM, Hein DW, Zheng W (2008) Meat intake, heterocyclic amine exposure, and metabolizing enzyme polymorphisms in relation to colorectal polyp risk. Cancer Epidemiol Biomarkers Prev 17(2):320-329. doi:10.1158/ 1055-9965.EPI-07-0615

20. Martinez ME, Jacobs ET, Ashbeck EL, Sinha R, Lance P, Alberts DS, Thompson PA (2007) Meat intake, preparation methods, mutagens and colorectal adenoma recurrence. Carcinogenesis 28(9):2019-2027. doi:10.1093/carcin/bgm179

21. Rohrmann S, Hermann S, Linseisen J (2009) Heterocyclic aromatic amine intake increases colorectal adenoma risk: findings from a prospective European cohort study. Am J Clin Nutr 89(5):1418-1424. doi:10.3945/ajen.2008.26658

22. Ferrucci LM, Sinha R, Graubard BI, Mayne ST, Ma X, Schatzkin A, Schoenfeld PS, Cash BD, Flood A, Cross AJ (2009) Dietary meat intake in relation to colorectal adenoma in asymptomatic women. Am J Gastroenterol 104(5):1231-1240. doi:10.1038/ajg. 2009.102

23. Wang H, Yamamoto JF, Caberto C, Saltzman B, Decker R, Vogt TM, Yokochi L, Chanock S, Wilkens LR, Le Marchand L (2010) Genetic variation in the bioactivation pathway for polycyclic hydrocarbons and heterocyclic amines in relation to risk of colorectal neoplasia. Carcinogenesis 32(2):203-209. doi:10.1093/ carcin/bgq237

24. Rushmore TH, Kong AN (2002) Pharmacogenomics, regulation and signaling pathways of phase I and II drug metabolizing enzymes. Curr Drug Metab 3(5):481-490

25. Sinha R, Rothman N, Brown ED, Mark SD, Hoover RN, Caporaso NE, Levander OA, Knize MG, Lang NP, Kadlubar FF (1994) Pan-fried meat containing high levels of heterocyclic aromatic amines but low levels of polycyclic aromatic hydrocarbons induces cytochrome P4501A2 activity in humans. Cancer Res 54(23):6154-6159
26. Turesky RJ (2004) The role of genetic polymorphisms in metabolism of carcinogenic heterocyclic aromatic amines. Curr Drug Metab 5(2):169-180

27. Eichholzer M, Rohrmann S, Barbir A, Hermann S, Teucher B, Kaaks R, Linseisen J (2012) Polymorphisms in heterocyclic aromatic amines metabolism-related genes are associated with colorectal adenoma risk. Int $\mathrm{J}$ Mol Epidemiol Gen (accepted)

28. Ferrucci LM, Cross AJ, Gunter MJ, Ahn J, Mayne ST, Ma X, Chanock SJ, Yeager M, Graubard BI, Berndt SI, Huang WY, Hayes RB, Sinha R (2010) Xenobiotic metabolizing genes, meatrelated exposures, and risk of advanced colorectal adenoma. World Rev Nutr Diet 101:34-45. doi:10.1159/000314509

29. Hainaut P, Vozar B, Rinaldi S, Riboli E, Caboux E (2011) The European prospective investigation into cancer and nutrition biobank. Methods Mol Biol 675:179-191. doi:10.1007/9781-59745-423-0_7

30. Riboli E, Hunt KJ, Slimani N, Ferrari P, Norat T, Fahey M, Charrondiere UR, Hemon B, Casagrande C, Vignat J, Overvad K, Tjonneland A, Clavel-Chapelon F, Thiebaut A, Wahrendorf J, Boeing H, Trichopoulos D, Trichopoulou A, Vineis P, Palli D, Bueno-De-Mesquita HB, Peeters PH, Lund E, Engeset D, Gonzalez CA, Barricarte A, Berglund G, Hallmans G, Day NE, Key TJ, Kaaks R, Saracci R (2002) European Prospective Investigation into Cancer and Nutrition (EPIC): study populations and data collection. Public Health Nutr 5(6B):1113-1124. doi: 10.1079/PHN2002394

31. Bohlscheid-Thomas S, Hoting I, Boeing H, Wahrendorf J (1997) Reproducibility and relative validity of food group intake in a food frequency questionnaire developed for the German part of the EPIC project. European Prospective Investigation into Cancer and Nutrition. Int J Epidemiol 26(Suppl 1):S59-S70

32. Rohrmann S, Zoller D, Hermann S, Linseisen J (2007) Intake of heterocyclic aromatic amines from meat in the European Prospective Investigation into Cancer and Nutrition (EPIC)-Heidelberg cohort. Br J Nutr 98(6):1112-1115

33. Skog K, Augustsson K, Steineck G, Stenberg M, Jagerstad M (1997) Polar and non-polar heterocyclic amines in cooked fish and meat products and their corresponding pan residues. Food Chem Toxicol 35(6):555-565

34. Sinha R, Rothman N, Salmon CP, Knize MG, Brown ED, Swanson CA, Rhodes D, Rossi S, Felton JS, Levander OA (1998) Heterocyclic amine content in beef cooked by different methods to varying degrees of doneness and gravy made from meat drippings. Food Chem Toxicol 36(4):279-287

35. Nordmark A, Lundgren S, Ask B, Granath F, Rane A (2002) The effect of the CYP1A2 *1F mutation on CYP1A2 inducibility in pregnant women. Br J Clin Pharmacol 54(5):504-510

36. Butler LM, Duguay Y, Millikan RC, Sinha R, Gagne JF, Sandler RS, Guillemette C (2005) Joint effects between UDP-glucuronosyltransferase 1A7 genotype and dietary carcinogen exposure on risk of colon cancer. Cancer Epidemiol Biomarkers Prev 14(7):1626-1632. doi:10.1158/1055-9965.EPI-04-0682

37. Engelke CE, Meinl W, Boeing H, Glatt H (2000) Association between functional genetic polymorphisms of human sulfotransferases 1A1 and 1A2. Pharmacogenetics 10(2):163-169

38. Magagnotti C, Pastorelli R, Pozzi S, Andreoni B, Fanelli R, Airoldi L (2003) Genetic polymorphisms and modulation of 2-amino-1-methyl-6-phenylimidazo[4,5-b]pyridine (PhIP)-DNA adducts in human lymphocytes. Int J Cancer 107(6):878-884. doi:10.1002/ijc.11492

39. Yamanaka H, Nakajima M, Katoh M, Hara Y, Tachibana O, Yamashita J, McLeod HL, Yokoi T (2004) A novel polymorphism in the promoter region of human UGT1A9 gene (UGT1A9*22) and its effects on the transcriptional activity. Pharmacogenetics 14(5):329-332 
40. Hein DW, Doll MA, Fretland AJ, Leff MA, Webb SJ, Xiao GH, Devanaboyina US, Nangju NA, Feng Y (2000) Molecular genetics and epidemiology of the NAT1 and NAT2 acetylation polymorphisms. Cancer Epidemiol Biomarkers Prev 9(1):29-42

41. Le Marchand L, Hankin JH, Pierce LM, Sinha R, Nerurkar PV, Franke AA, Wilkens LR, Kolonel LN, Donlon T, Seifried A, Custer LJ, Lum-Jones A, Chang W (2002) Well-done red meat, metabolic phenotypes and colorectal cancer in Hawaii. Mutat Res 506-507:205-214

42. Cotterchio M, Boucher BA, Manno M, Gallinger S, Okey AB, Harper PA (2008) Red meat intake, doneness, polymorphisms in genes that encode carcinogen-metabolizing enzymes, and colorectal cancer risk. Cancer Epidemiol Biomarkers Prev 17(11):3098-3107. doi:10.1158/1055-9965.EPI-08-0341

43. Lilla C, Risch A, Verla-Tebit E, Hoffmeister M, Brenner H, Chang-Claude J (2007) SULT1A1 genotype and susceptibility to colorectal cancer. Int J Cancer 120(1):201-206. doi:10.1002/ijc. 22156

44. Turesky RJ, Vouros P (2004) Formation and analysis of heterocyclic aromatic amine-DNA adducts in vitro and in vivo. J Chromatogr B Analyt Technol Biomed Life Sci 802(1): 155-166. doi:10.1016/j.jchromb.2003.10.053

45. Nguyen TV, Janssen MJ, van Oijen MG, Bergevoet SM, te Morsche RH, van Asten H, Laheij RJ, Peters WH, Jansent JB
(2010) Genetic polymorphisms in GSTA1, GSTP1, GSTT1, and GSTM1 and gastric cancer risk in a Vietnamese population. Oncol Res 18(7):349-355

46. Sweeney C, Coles BF, Nowell S, Lang NP, Kadlubar FF (2002) Novel markers of susceptibility to carcinogens in diet: associations with colorectal cancer. Toxicology 181-182:83-87

47. Turesky RJ, Le Marchand L (2011) Metabolism and biomarkers of heterocyclic aromatic amines in molecular epidemiology studies: lessons learned from aromatic amines. Chem Res Toxicol 24(8):1169-1214. doi:10.1021/tx200135s

48. Tijhuis MJ, Wark PA, Aarts JM, Visker MH, Nagengast FM, Kok FJ, Kampman E (2005) GSTP1 and GSTA1 polymorphisms interact with cruciferous vegetable intake in colorectal adenoma risk. Cancer Epidemiol Biomarkers Prev 14(12):2943-2951. doi: 10.1158/1055-9965.EPI-05-0591

49. Skjelbred CF, Saebo M, Hjartaker A, Grotmol T, Hansteen IL, Tveit KM, Hoff G, Kure EH (2007) Meat, vegetables and genetic polymorphisms and the risk of colorectal carcinomas and adenomas. BMC Cancer 7:228. doi:10.1186/1471-2407-7-228

50. Tiemersma EW, Wark PA, Ocke MC, Bunschoten A, Otten MH, Kok FJ, Kampman E (2003) Alcohol consumption, alcohol dehydrogenase 3 polymorphism, and colorectal adenomas. Cancer Epidemiol Biomarkers Prev 12(5):419-425 